\title{
Clinical Reasoning: A 63-year-old man with gastroenteritis progressing to stupor and quadriparesis
}

Asya I. Wallach, MD, Ilya Kister, MD, and Eytan Raz, MD, PhD

Neurology ${ }^{\circledR}$ 2020;94:e1107-e1111. doi:10.1212/WNL.0000000000009057
Correspondence

Dr. Wallach

AsyaWallach@gmail.com.

\section{Section 1}

A 63-year-old man with a history of chronic right-sided headaches and intermittent nausea presented to the emergency department with worsening headache, nausea, and nonstop vomiting for 12 hours.

Headache history dated back to the patient's early 20s. Headaches were exclusively right-sided, throbbing, associated with right eye and neck pain, photophobia and osmophobia, but without autonomic features. They occurred several times per week. He underwent multiple dental procedures, chiropractic manipulations, and C2-3 discectomy, but these interventions did not provide lasting relief, and he relied on nonsteroidal anti-inflammatory drugs to control the pain. Four years prior to his current hospital admission, he started to experience lightheadedness on getting up that resolved with lying down. Two years prior, he noticed problems with balance and had several falls. Six months prior, he had profound nausea for a week and lightheadedness that prevented him from getting out of bed. The symptoms abated on their own, but he would wake up daily with mild nausea and lightheadedness ever since. His medical history was otherwise only significant for asthma, hypertension, and obstructive sleep apnea.

On admission, the patient was diagnosed with gastroenteritis and treated with fluids and antiemetics. The vomiting stopped, but the headache continued, and he developed new neurologic signs over several days: diplopia on left gaze, weakness progressing to quadriparesis, and increasing unresponsiveness (family described him as being "completely out of it—comatose").

\section{Questions for consideration:}

1. What is the localization of these signs and symptoms?

2. What diagnostic tests would you perform now?

GO TO SECTION 2 


\section{Section 2}

The combination of persistent vomiting, diplopia, quadriparesis, and altered sensorium can localize to the brainstem. Area postrema in the dorsal medulla oblongata is a chemoreceptor zone for emesis. Binocular diplopia in this case most likely localizes to the midbrain or pons, but may also be due to a lesion anywhere along cranial nerves 3, 4 , and 6. Altered sensorium may be due to disruption of the ascending reticular activating system (brainstem tegmentum lesion or bilateral diencephalic lesions). Quadriparesis may result from a lesion affecting the bilateral corticospinal tracts at the level of the brainstem or cervical spine. Another possibility is a large supratentorial lesion causing herniation and increased intracranial pressure, but this presentation would be expected to cause papilledema.

Given the differential, emergent neuroimaging of the brain and upper cervical spine was performed. MRI of the brain demonstrated extensive hyperintense T2/fluid-attenuated inversion recovery signal within the inferior pons and the medulla with patchy contrast enhancement on T1-weighted sequences throughout the brainstem and the cerebellum (figure). The cervical cord was normal.

\section{Questions for consideration:}

1. What is the differential diagnosis for a partially enhancing, multifocal, posterior fossa lesion?

2. How would you manage the patient at this point?

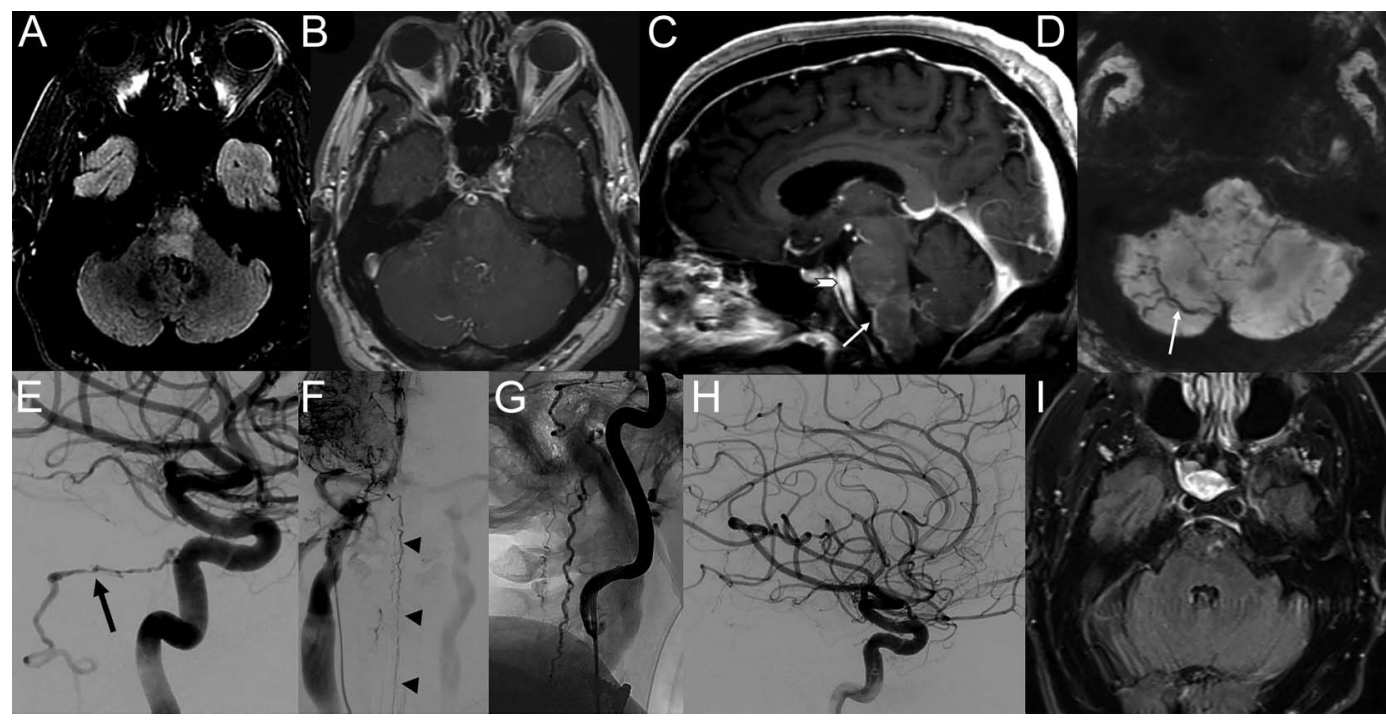

(A, B) Initial axial MRI brain at the level of the pons demonstrates pontine fluid-attenuated inversion recovery (FLAIR) hyperintensity with patchy enhancement. (C) Sagittal T1 postcontrast image demonstrates enhancement at the level of the inferior pons, medulla, and cerebellar white matter. It also shows an enlarged anterior pontomedullary vein (arrow) distinguishable from the basilar artery, which is more anterior (arrowhead). (D) Axial susceptibility-weighted imaging demonstrates increased size and tortuosity of bilateral, right greater than left, pial cerebellar veins (arrow); there are also a few microhemorrhages in the cerebellum and brainstem. (E-G) Diagnostic angiogram, right internal carotid artery injection. (E) Subtracted lateral view demonstrates increased size of the meningohypophyseal trunk arising from the posterior cavernous segment of the internal carotid artery, with a change in caliber (arrow) corresponding to the location of the dural arteriovenous fistula along the right petrous ridge. The venous outflow is in the inferior direction, toward the petrous vein and the anterior pontomedullary vein (arrowheads), all the way down to the upper thoracic spine as shown in (F) on the frontal view, and (G) on the lateral view. (H) Subtracted lateral view from right internal carotid artery injection after endovascular occlusion of the dural arteriovenous fistula using n-butyl cyanoacrylate. The fistula has been cured. (I) One month postprocedure, axial FLAIR at the level of the pons demonstrates near complete resolution of the previously seen abnormal signal. 


\section{Section 3}

The differential diagnosis for a large, partially enhancing, T2hyperintense lesion centered around the pons and medulla includes inflammatory, granulomatous, and paraneoplastic conditions such as multiple sclerosis, neuromyelitis optica, myelin oligodendrocyte glycoprotein (MOG) antibody syndrome, neuro-Behçet, Bickerstaff brainstem encephalitis, glial fibrillary acidic protein astrocytopathy, chronic lymphocytic inflammation with pontine perivascular enhancement responsive to steroids (CLIPPERS), hemophagocytic lymphohistiocytosis, neurosarcoidosis, and $\gamma$-aminobutyric acid-B receptor encephalitis; acute and chronic infectious diseases, such as varicella, tuberculosis, neurosyphilis, Listeria monocytogenes, and Whipple disease; and neoplastic and hematologic disorders, including CNS lymphoma, intravascular lymphoma, systemic T-cell lymphoma, Hodgkin lymphoma, CNS lymphomatoid granulomatosis, and Langerhans cell histiocytosis.

Our patient had no history of neoplastic or rheumatologic disease, and no findings pointing to a multisystem disorder (no systemic symptoms; no rash, ulcers, or joint swelling). Laboratory testing, which included complete blood count, comprehensive metabolic panel, antinuclear antibodies, dsDNA, angiotensin-converting enzyme, Rf, Scl70, antineutrophil cytoplasmic antibodies, serum paraneoplastic panel, neuromyelitis optica and MOG antibodies, erythrocyte sedimentation rate, $\mathrm{C}$-reactive protein, ferritin, thyroidstimulating hormone, vitamin $\mathrm{B}_{6}$ and vitamin $\mathrm{B}_{12}$, serum and urine protein electrophoresis, and syphilis, Lyme disease, and hepatitis serologies, were unremarkable. CSF analysis revealed normal cells, protein, and glucose, absent oligoclonal bands, normal immunoglobulin $\mathrm{G}$ index, and negative bacterial, mycobacterial, fungal, spirochetal, and viral studies. CT of the body showed a thyroid nodule and a cyst in the liver. PET CT demonstrated increased signal uptake at the thyroid nodule.

Given the negative workup, the leading diagnosis was CLIPPERS, which can present as a subacute pontocerebellar syndrome associated with multiple contrast-enhancing nodules centered at the pons. ${ }^{1}$ Lesions in CLIPPERS are typically small $(<3 \mathrm{~mm})$ and show neither mass effect nor ring enhancement. The area of $\mathrm{T} 2$ hyperintensity does not usually extend beyond the area of enhancement. Pathology demonstrates a dense, T-cell lymphocytic-predominant inflammation, predominantly perivascular, with diffuse infiltration of the gray and white matter, but without loss of myelin. Clinical symptoms and contrast enhancement on MRI improve with corticosteroid therapy. ${ }^{2}$ Thus our patient was treated initially with pulse IV methylprednisolone. The patient appeared more awake to his family with gradual clinical improvement over the course of 1 week. MRI brain did not demonstrate a substantial radiographic improvement following IV steroids. Upon discharge, the patient's balance remained impaired and he required a walker. He was instructed to continue oral prednisone and follow up in the outpatient neurology clinic.

\section{Questions for consideration:}

1. Which clinical and radiologic features of this case would be atypical for CLIPPERS?

2. What additional diagnostic possibilities should be considered?

3. What studies are indicated at this point?

GO TO SECTION 4 


\section{Section 4}

Clinical symptoms and MRI lesions due to CLIPPERS are expected to respond to steroids, at least temporarily. Although our patient had improved after steroids, the response was incomplete and there was no resolution of the enhancing lesions, which is inconsistent with CLIPPERS. A careful review of the MRI showed that the rather diffuse area of T2 hyperintensity was considerably larger than the area of enhancement, which is atypical for CLIPPERS. Additional unusual radiographic features noted were increased size and tortuosity of the right pial cerebellar veins and microhemorrhages (figure). These findings raised the possibility of venous congestion. CT angiogram showed an enlarged tortuous venous vessel along the ventral surface of the brainstem (visible also on postcontrast MRI; figure), raising suspicion for the presence of an intracranial dural arteriovenous fistula (dAVF) with drainage into the perimedullary venous system. A cerebral angiogram was performed, which confirmed the presence of a right petrous ridge dAVF supplied by the meningohypophyseal trunk, a branch of the proximal cavernous segment of the internal carotid artery, with venous outflow into the anterior pontomesencephalic vein (longitudinally towards the cervicothoracic spine) (figure). This finding led to a reinterpretation of the patient's clinical and radiographic findings. His longstanding headaches, stepwise progression of neurologic symptoms, and the dramatic brainstem presentation with quadriparesis and stupor on the latest hospital admission could all plausibly be attributed to severe brainstem and cerebellar venous congestion from a dAVF. The patient was therefore treated with endovascular occlusion of the dAVF using transarterial n-butyl cyanoacrylate glue (figure). He tolerated the procedure well with the exception of ischemia to cranial nerve VI, resulting in a partial sixth nerve palsy. One month postprocedure, his MRI showed significant improvement (figure) in lesion size and he was tapered off prednisone. At 2-month follow-up, the patient reported his symptoms had significantly improved such that he had returned to work and was ambulating with a cane, and his diplopia had resolved. He still had headaches, but not as severe as before, and well-controlled with nonsteroidal antiinflammatory drugs.

\section{Discussion}

We present a case of an intracranial dAVF masquerading as CLIPPERS. dAVF is a rare vascular malformation, accounting for $10 \%-15 \%$ of intracranial vascular malformations, ${ }^{3}$ with an incidence of 0.16 per 100,000 adults per year. ${ }^{4}$ Still rarer is an intracranial dAVF with spinal venous drainage (Cognard type $\mathrm{V}$ arteriovenous fistula). ${ }^{5,6}$ What makes our case even more unusual is that type $\mathrm{V}$ dAVF typically presents with progressive myelopathy or subarachnoid hemorrhage, while our patient presented with an acute brainstem syndrome. Congestion with hemorrhage and ischemia secondary to intracranial dAVF is a very rare presentation, with fewer than 40 cases reported to date. ${ }^{5,7}$ Choice of treatment-endovascular or neurosurgical — depends on the specific vascular anatomy of the dAVF. If these approaches are unavailable or unsuccessful, stereotactic radiosurgery may be an option. ${ }^{8}$ Steroids are not recommended for dAVF as there are reports of acute clinical worsening, presumably due to mineralocorticoid-induced increased venous pressure. ${ }^{9,10}$

In a patient with new-onset headache or chronic side-locked headaches with emerging neurologic symptoms, the possibility of a secondary headache must be considered and MRI brain is indicated. In our case, the MRI brain demonstrated patchy contrast enhancement throughout the brainstem and cerebellum on the postcontrast scan and enlarged vessels prompting a closer evaluation of the cerebral and spinal vasculature with an angiogram. dAVF in the petrous ridge may cause tinnitus, which needs to be asked about directly, and a cranial bruit may be auscultated on examination.

Intracranial dAVF with brainstem congestion is very rare, but it must be considered in the differential diagnosis of CLIPPERS and other contrast-enhancing brainstem lesions because timely intervention could prevent a devastating outcome (severe disability or death). To our knowledge, intracranial dAVF misdiagnosed as CLIPPERS has not been previously reported.

\section{Study funding}

No targeted funding reported.

\section{Disclosure}

The authors report no disclosures relevant to the manuscript. Go to Neurology.org/N for full disclosures.

\begin{tabular}{llll}
\multicolumn{2}{l}{ Appendix } & Authors \\
\hline Name & Location & Role & Contribution \\
\hline $\begin{array}{l}\text { Asya I. } \\
\text { Wallach, } \\
\text { MD }\end{array}$ & $\begin{array}{l}\text { New York } \\
\text { University School } \\
\text { of Medicine, New } \\
\text { York }\end{array}$ & Author & $\begin{array}{l}\text { Drafting the manuscript, } \\
\text { study concept and design, } \\
\text { analysis and acquisition of } \\
\text { the data }\end{array}$ \\
\hline $\begin{array}{l}\text { Ilya } \\
\text { Kister, } \\
\text { MD }\end{array}$ & $\begin{array}{l}\text { New York } \\
\text { University School }\end{array}$ & Author & $\begin{array}{l}\text { Revising the manuscript for } \\
\text { content, analysis and } \\
\text { interpretation of data, study } \\
\text { supervision }\end{array}$ \\
\hline $\begin{array}{l}\text { Eytan } \\
\text { Raz, MD, } \\
\text { PhD }\end{array}$ & $\begin{array}{l}\text { New York } \\
\text { University School } \\
\text { of Medicine, New } \\
\text { York }\end{array}$ & Author & $\begin{array}{l}\text { Revising the manuscript for } \\
\text { content, analysis and } \\
\text { interpretation of data, study } \\
\text { supervision }\end{array}$ \\
\hline
\end{tabular}

\section{References}

1. Tobin WO, Guo Y, Krecke KN, et al. Diagnostic criteria for chronic lymphocytic inflammation with pontine perivascular enhancement responsive to steroids (CLIPPERS). Brain 2017;140:2415-2425.

2. Zalewski NL, Tobin WO. CLIPPERS. Curr Neurol Neurosci Rep 2017;17:65.

3. Kwon BJ, Han MH, Kang HS, Chang KH. MR imaging findings of intracranial dural arteriovenous fistulas: relations with venous drainage patterns. AJNR Am J Neuroradiol 2005;26:2500-2507.

4. Al-Shahi R, Bhattacharya JJ, Currie DG, et al. Prospective, population-based detection of intracranial vascular malformations in adults: the Scottish Intracranial Vascular Malformation Study (SIVMS). Stroke 2003;34:1163-1169. 
5. Satoh M, Kuriyama M, Fujiwara T, Tokunaga K, Sugiu K. Brainstem ischemia from intracranial dural arteriovenous fistula: case report. Surg Neurol 2005;64:341-345.

6. Gomez J, Amin AG, Gregg L, Gailloud P. Classification schemes of cranial dural arteriovenous fistulas. Neurosurg Clin N Am 2012;23:55-62.

7. Copelan AZ, Krishnan A, Marin H, Silbergleit R. Dural arteriovenous fistulas: a characteristic pattern of edema and enhancement of the medulla on MRI. AJNR Am J Neuroradiol 2018;39:238-244.
8. Chen CJ, Lee CC, Ding D, et al. Stereotactic radiosurgery for intracranial dural arteriovenous fistulas: a systematic review. J Neurosurg 2015;122:353-362.

9. Takahashi H, Ueshima $\mathrm{T}$, Goto $\mathrm{D}$, et al. Acute tetraparesis with respiratory failure after steroid administration in a patient with a dural arteriovenous fistula at the craniocervical junction. Intern Med 2018;57:591-594.

10. Ma Y, Zhang H, Lu J, Wang X, Jiao L, Ling F. Glucocorticoid induced acute paraplegia in a patient with intracranial dural arteriovenous fistula. Neurol Sci 2019;40:2411-2414. 


\section{Neurology}

\section{Clinical Reasoning: A 63-year-old man with gastroenteritis progressing to stupor and quadriparesis}

Asya I. Wallach, Ilya Kister and Eytan Raz

Neurology 2020;94;e1107-e1111 Published Online before print February 25, 2020

DOI 10.1212/WNL.0000000000009057

This information is current as of February 25, 2020

\section{Updated Information \&} Services

References

Subspecialty Collections

Permissions \& Licensing

Reprints including high resolution figures, can be found at: http://n.neurology.org/content/94/10/e1107.full

This article cites 10 articles, 2 of which you can access for free at: http://n.neurology.org/content/94/10/e1107.full\#ref-list-1

This article, along with others on similar topics, appears in the following collection(s):

All Demyelinating disease (CNS)

http://n.neurology.org/cgi/collection/all_demyelinating_disease_cns Secondary headache disorders

http://n.neurology.org/cgi/collection/secondary_headache_disorders

Information about reproducing this article in parts (figures,tables) or in its entirety can be found online at:

http://www.neurology.org/about/about_the_journal\#permissions

Information about ordering reprints can be found online:

http://n.neurology.org/subscribers/advertise

Neurology ${ }^{\circledR}$ is the official journal of the American Academy of Neurology. Published continuously since 1951, it is now a weekly with 48 issues per year. Copyright @ 2020 American Academy of Neurology. All rights reserved. Print ISSN: 0028-3878. Online ISSN: 1526-632X.

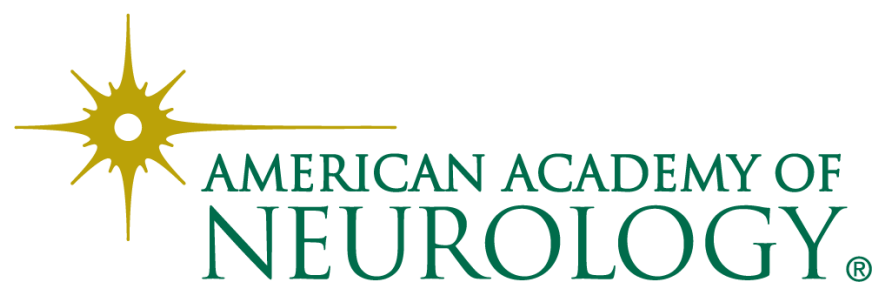

